\title{
Are inductively derived categories of facial activity reliable when applied to high-risk infants tested before term age?
}

\author{
THOMAS E. HANNAN and ADRIANA E. MOLITOR \\ Duke University, Durham, North Carolina
}

\begin{abstract}
The techniques of facial coding employed with full-term infants have been applied to studies of premature infants tested before 40 weeks postconceptional age. The purpose of this research was to evaluate the methodological wisdom of using the coding strategies that were developed for older populations of infants with high-risk, premature infants who are tested while still in the hospital. As a first step, we tested the eight most commonly used categories of facial activity. The results were not encouraging; the mean $r(\beta)$ was .61 and the mean $\kappa$ was .52. On the basis of our assessment of the limitations in using these traditional facial categories, we developed six new categories of action. This time the results were more positive; the mean $r(\beta)$ was .92 and the mean $\kappa$ was .80 . The new catalog appears to be a reliable representation of a range of facial behaviors observed in infants who are tested well before term age.
\end{abstract}

Inductively derived systems for coding the behavior of humans have been developed using ethological observational methods to derive ethograms or catalogs of apparently meaningful regularities of behavior. Coding systems employing inductively derived facial categories have facilitated the exploration of numerous facets of the socioemotional and sociocommunicative development of young, full-term infants (see, e.g., Boccia \& Campos, 1989; Brazelton, Koslowski, \& Main, 1974; Field, Vega-Lahr, Goldstein, \& Scafidi, 1987; Kaye \& Fogel, 1980; Malatesta, Grigoryev, Lamb, Albin, \& Culver, 1986). Although the use of inductively derived categories of coding for the study of emotion has been criticized, their use in studies of sociocommunicative processes has not (see Campos, Barrett, Lamb, Goldsmith, \& Sternberg, 1983). Ultimately, the level of measurement of the process reflected by the rhythm and flow of interaction should be reasonably matched to the categorical data created from video records. In studies of sociocommunicative processes, it is important that the categories coded represent actions that are discernible by an adult interactant because it is this level

Portions of this paper were presented at the Society for Research in Child Development Biennial Meeting, April 1991, in Seattle. Preparation of this manuscript was made possible through a program project grant from NICHD to C. Eckerman (HD-21354). We thank A. Choi, E. Daughtrey, M. Gay, B. Jinnette, and A. Monroe for their assistance in the development and testing of the coding categories, and J. Oehler, C. Eckerman, and A. Grasso for commenting on earlier versions of this manuscript. We also thank the mothers and their infants, as well as the staff of the Duke University Medical Center's Intensive Care Nursery, for their cooperation during the collection of the data presented in this paper. Correspondence should be addressed to T. E. Hannan, P.O. Box 90539, Department of Psychology, Duke University, Durham, NC 27708-0539 (e-mail: thannan@acpub.duke.edu). of assessment that the adult is using while monitoring infant state during ongoing interaction.

The need to address the relationship of risk factors and the sociocommunicative competency of preterm infants has led to the use of categories of facial activity that were developed primarily for use with normal, full-term populations. Inductively derived categories of facial activity have been used in studies of preterm infants who were tested after term age (see, e.g., Bakeman \& Brown, 1980; Beckwith, Cohen, Kopp, Parmelee, \& Marcy, 1976; Malatesta, Tesman, \& Shepard, 1990; Minde, Perrotta, \& Marton, 1985) and prior to term age (see, e.g., Field, Woodson, Cohen, Greenberg, Garcia, \& Collins, 1983; Minde, Whitelaw, Brown, \& Fitzhardinge, 1983; Oehler, Eckerman, \& Wilson, 1988). Most recently, inductively derived categories of facial activity have been employed in studies of much younger, higher biological risk infants who were tested as much as 12 weeks before normal term (e.g., Eckerman \& Oehler, 1992; Eckerman, Oehler, Hannan, \& Molitor, 1995; Eckerman, Oehler, Medvin, \& Hannan, 1994).

It is appropriate to ask whether the strategies of coding inductively derived categories of facial activity that have evolved for use with full-term infants are reliable when applied to very young preterm infants, and especially preterm infants at higher levels of biological risk. A number of factors suggest that there may be limitations to the application of inductively derived strategies for coding the facial activity of higher biological risk populations of preterm infants. First, depending on the postconceptional age of the infant, the coordination of the motor elements that constitute each of the discrete expressions may not yet be part of the infant's repertoire of facial activity. Expressions are not restricted to one specific region of the face, but involve components from each 
of the major facial regions. The individual components may be apparent, but they may not display the same synergistic action as a more mature expression. Moreover, because of possible neurological, anatomical, or physiological dysfunctions, these infants may not display the same patterns of facial behavior as normal, full-term infants. In addition, task constraints (e.g., the gravitational pull on the facial musculature while the infant is in the upright position for testing, or the presence of appliances necessary for survival, such as cannulas or gavages) may restrict the muscles from forming certain discrete expressions or block a clear view of the infant's face. This last point is particularly relevant to studies of preterm infants in which the infants are tested while they are still in the intensive care nursery. Anyone who has observed very prematurely born infants in an intensive care nursery is well aware of the amount of tape that is used to secure appliances as nonintrusive as cannulas, let alone the amount of tape that is used to secure appliances as intrusive as oral or nasal gavage tubes. In some cases, the entire mouth is obscured by tape; in other cases, the corners of the mouth and the cheeks are obscured by the tape.

These methodological constraints do not exist with fullterm infants or preterm infants who are tested after release from the hospital, thus making them easier to test than very prematurely born infants. We are faced with a number of choices regarding prematurely born infants: First, we could simply choose not to test infants at this age (thus avoiding an opportunity to understand how these infants first present as social partners). Second, we could decide that we would test only those infants who do not need any type of medical support, thus eliminating from our sample the infants that are categorized as being at highest biological risk (leaving us with very little generalizability to the population of very low birthweight infants and little understanding of how risk interacts with prematurity in the earliest social encounters). Third, we can accept the methodological problems associated with this population and use the best possible strategies for examining their earliest behavior. We have opted for the third choice.

The purpose of this paper is to report the results of a systematic examination of the reliability of a set of inductively derived categories of facial activity that were applied to a sample of preterm infants who were videotaped during the weeks prior to term age. We are not addressing the issue of the validity of using inductively derived categories as an index of state, nor are we addressing the issue of the comparative validity of inductively derived, anatomically comprehensive (see, e.g., Eckman \& Friesen, 1978; Oster, 1978; Oster \& Rosenstein, in press) or templatebased (see, e.g., Izard, 1979; Izard \& Dougherty, 1980) strategies of representing the face. For studies of social interaction, where the emphasis is on "social states" composed of indices of separate regions of infant activity, inductively derived categories seem to provide a reasonable sample of infant behavior. We acknowledge that for studies of the development of affect or the evolution of facial expressions, inductively derived categories would not pro- vide a reasonable sample of infant behavior. In the present case, however, we are addressing the issue of whether the existing inductively derived categories of facial activity, which evolved through work with full-term infants, can be directly applied to all classes of preterm infants tested weeks prior to term age.

\section{EXPERIMENT 1}

\section{Method}

The data for this paper were chosen from a project examining the socioemotional development of preterm infants from 29 weeks postconceptional age to term age. Details of the subject recruitment and testing are available elsewhere (cf. Eckerman \& Oehler, 1992; Eckerman et al., 1995; Eckerman et al., 1994).

The sample was composed of infants who met the following criteria: (1) inborn at Duke Unıversity Medical Center, (2) birthweight less than $1,501 \mathrm{~g}$, (3) absence of major congenital defects, (4) maternal residence within a 50-mile radius of Durham County (to enable a follow-up), and (5) maternal consent to participate in the study. The mean gestational age of the subjects was 29 weeks; $54 \%$ were first born; $53 \%$ fell into Categories 4 and 5 of the Hollingshead two-factor index (Hollingshead, 1957); 59\% were black; and $51 \%$ were females.

All tests were conducted in the Duke University Medical Center Intensive Care Nursery. Testing began once the infant was at least 29 weeks postconceptional age and determined to be medically stable. For each test, the infant was removed from the isolette and placed in an adjacent infant seat. All life support apparatus (e.g., intravenous lines, central lines, cannulas, gavage tubes, etc.), as well as neonatal monitoring sensors, were left undisturbed. Testing occurred biweekly until the infant was 37 weeks. Each of the tests presented $4.5 \mathrm{~min}$ of standardized social stimulation (talking and touching by a trained examiner), and each test was videotaped.

The videotaped sessions were coded via the Observational Coding System (OCS) Tools (Triangle Research Collaborative, 1985), a software and hardware data entry system linking the computer to a videocassette recorder. OCS Tools places a tıme signal (accurate to $.01 \mathrm{sec}$ ) on one audio track of the videotape. This permanent digital time signal is read by the computer during coding, allowing each videotaped session to be coded into a stream of mutually exclusive categories of action the onsets of which were associated with a continuous time line. Observers were trained to note changes in facial activity and assign the appropriate category of activity that followed the detection of a change. Accuracy in onset and offset detection was ensured by having the coders observe a change, note the new category, then rewind the tape to the exact place where the change took place and record the event via a computer keystroke. The exact time of the event was automatically entered each time a computer keystroke occurred. In addition, observers were encouraged to rewind the tape and observe sections again if they were having difficulty distinguishing the category of activity.

To be conservative in our reliability assessments, we decided to calculate both Cohen's $\kappa$ (Cohen, 1960) and Kaye's $r(\beta)$ (Kaye, 1980 ) and to base our decisions on the convergence of the two values. Cohen's $\kappa$ is composed of the value $P_{O}$ (the observed proportion of agreements) and $P_{C}$ (the chance proportion of agreements):

$$
\kappa=\frac{\left(P_{O}-P_{C}\right)}{\left(1-P_{C}\right)}
$$

We employed the techniques for applying $\kappa$ to continuous time data that have been described by Hollenbeck (1978). Diagonal entries in the $\kappa$ matrix record the amount of time that agreement occurred, while off-diagonal values record the amount of time that disagreements occurred as well as the latency or time lag between detection 
Table 1

Coding Categories Employed in the Initial Assessment

\begin{tabular}{|c|c|}
\hline Category & Description of Category \\
\hline cry-face & $\begin{array}{l}\text { The infant's eyes are tightly closed, brow furrowed, } \\
\text { mouth open wide with tongue often protruding. } \\
\text { Code offset of cry-face when face has completely } \\
\text { returned to a relaxed expression or other codable } \\
\text { expressions. }\end{array}$ \\
\hline distress & $\begin{array}{l}\text { Code only unquestionable distressed faces. Facial } \\
\text { distortion is evidenced by the infant's mouth re- } \\
\text { tracting slightly; the cheeks go up and the eyes, } \\
\text { nose, and cheek area are "scrunched" together so } \\
\text { that the eyes look tightly squinted. The nose is } \\
\text { wrinkled and the cheeks are tight and slightly } \\
\text { lifted. }\end{array}$ \\
\hline
\end{tabular}

grimace/disgust Infant's mouth is retracted to an extreme. The cheeks remain more or less in their usual position; they do not move in as noticeably as in the distress face. There is a marked asymmetry to the face and the infant is displaying repetitive tongue protrusions that suggest a bad taste in the mouth.

relaxed There is no tension in the face.

yawn The infant's eyes are usually shut and squinted; the mouth opens very wide with a deep inspiration of air (chest may visibly expand, and legs and toes may rise as yawn begins). It is common to see infants "try to yawn" before completing an actual yawn (it may appear to be a "sigh"). Do not code these attempts or false starts as yawns. Begin coding the yawn from the start of the yawn face (mouth begins to open) through the complete resolution of the yawn (the face usually forms a distressed or grimaced look before returning to a relaxed state).

smile The corners of the infant's mouth turn upward either slightly or more broadly as in a full smile. Eyes may widen and brighten and some brow lifting and forehead wrinkling may be present. Do not code slight or "incipient" smiles.

mouthing Lip movements appear as if the infant is "talking." This behavior cannot occur with any negative facial tone. Infant may repetitively protrude the tongue, but the face must be neutral. Include chewing, lip smacking, and bubble blowing.

sucking Infant is sucking while pacifier is in the mouth, or pacifier is in the mouth and it is not clear if any other mouth or face activities are on. If the infant has the pacifier in the mouth and the cry-face, distressed face, smile, or mouthing occurs, use these codes instead of sucking.

of an event by one coder versus the second coder. The value of $\kappa$ is the ability to assess agreement once chance agreement has been factored out. One weakness in the $\kappa$ statistic is the reliance on temporal overlap without concern for onset and offset accuracy.

In contrast, Kaye's $r(\beta)$ is an estimate of reliability that is composed of agreements and disagreements, with disagreements in turn composed of what Kaye called false alarms (one coder saw an event that the other did not) and missed events (events that occurred but neither coder detected them). To estimate $r(\beta)$, five basic components are derived: $J$ (the number matches or joint agreements), $D$ (the number of nonmatches or disagreements), $F$ (the total number of events coded by the two observers or $D+2 J$ ), $T$ (the total time of the coded segment), and $t$ (the time tolerance or allowable lag for the determination of a potential match between the events identified by the first and second coders). The calculation of $r(\beta)$ results from the following equation:

$$
r(\beta)=1-D / F
$$

where $D / F$ results from the following equation:

$$
D / F=\left(2 \beta-\beta^{2}\right) e^{\left[-\left(2 \beta-\beta^{2}\right) F t T\right]} .
$$

The value of $r(\beta)$ is the reliance on agreement in onset and offset for determining a match, and the role that segment density plays in the coding process. For example, it should be easier to detect five events in a period of $10 \mathrm{~min}$ than it would be to detect 100 events in the same time period. Kraemer (1982) has criticized $r(\beta)$ because of its reliance on a Poisson distribution for estimating $\beta$, and her view is that the assumptions underlying the statistic are valid only if a standard can be used for comparison.

Because each measure has strengths and weaknesses, we decided to calculate both measures, and in line with Kraemer's (1982) concerns, we introduced a standard into the reliability assessments. We view as acceptable a minimum intercoder agreement of .70 for $\kappa$ and .80 for $r(\beta)$. In addition, we calculated the proportion of agreement by category to determine if there was an equal probability of each category being detected. To perform the calculations we used a variant of the reliability tests provided in the Micro-Analytic Data Analysis Package (Kienapple, 1987).

We first selected eight categories of facial expression to code: cry-face, distresis brow, grimace/disgust, relaxed, yawn, smile, mouthing, and sucking (Table 1). These categories had been used successfully in a number of prior studies of young full-term infants' social competencies (e.g., Fogel, 1979, 1981; Fogel \& Hannan, 1985; Hannan \& Fogel, 1987). Moreover, in all of the studies cited, the first author (T.H.) had the responsibility for training the coders. For the present assessment, training was conducted by the first author (T.H.) and consisted of approximately $60 \mathrm{~h}$ of practice coding. Following the training phase, each of the coders and the first author coded 12 randomly selected video segments that were evenly distributed across the 29-36 weeks postconceptional age period.

\section{Results and Discussion}

Table 2 displays the results of the reliability assessments. Observers A.C. and M.G. showed adequate agreement with each other on both the $r(\beta)$ and $\kappa$ measures; however, neither agreed as well with T.H. [T.H. provided a check on the accuracy of the application of the codes (see Hollenbeck, 1978) and served as the standard for calculation of the $r(\beta)$ values (see Kraemer, 1982)]. In only 1 of the 12 video segments was the mean $r(\beta)$ value greater than .70 , and in only 3 of the 12 video segments was the mean $\kappa$ value greater than .60 . Although it could be argued that the problem coder was T.H. (the standard), reference to the minimums (Min) and standard deviations ( $S D$ s) suggests that even though coders A.C. and M.G. appeared to be in agreement, there was a tremendous amount of variation across the 12 segments. Inspection of the $\kappa$ matrix and the mean proportion of agreement indicated that the agreement between A.C. and M.G.

Table 2

\begin{tabular}{|c|c|c|c|c|c|c|c|c|}
\hline & \multicolumn{2}{|c|}{ A.C./M.G. } & \multicolumn{2}{|c|}{ A.C./T.H. } & \multicolumn{2}{|c|}{ M.G./T.H. } & \multicolumn{2}{|c|}{ Totals } \\
\hline & $r(\beta)$ & $\kappa$ & $r(\beta)$ & $\kappa$ & $r(\beta)$ & $\kappa$ & $r(\beta)$ & $\kappa$ \\
\hline$N$ of cases & 12 & 12 & 12 & 12 & 12 & 12 & 12 & 12 \\
\hline$M$ & .78 & .71 & .48 & .39 & .56 & .45 & .61 & .52 \\
\hline Mdn & .84 & .73 & .46 & .39 & .57 & .47 & .61 & .50 \\
\hline$S D$ & .13 & .17 & .10 & .15 & .11 & .16 & .09 & .13 \\
\hline Min & .58 & .37 & .35 & .18 & .35 & .15 & .43 & .24 \\
\hline $\operatorname{Max}$ & 1.00 & .92 & .66 & .71 & .74 & .68 & .80 & .77 \\
\hline
\end{tabular}

Mean $r(\beta)$ and $\kappa$ Scores for the Initial Coding Catalog 
was higher because they were coding a smaller range of behavioral codes than was T.H., largely limited to yawn and relaxed face (mean proportion of agreement $=.95$ and .56, respectively). Further, it could be argued that the problem lay not with the codes, but with the training of the coders; however, the first author's previous experience with successfully training undergraduate coders at two other universities using the same codes would seem to greatly weaken this explanation (see Fogel, 1979, 1981; Fogel \& Hannan, 1985; Hannan \& Fogel, 1987). Although these alternative interpretations cannot be ruled out entirely, we think it most likely that the problem lies in the application of these coding categories to this population.

One difficulty that became apparent when we viewed the video sessions was the relative immaturity in the expressions displayed by these infants: In some cases, the expressions were not fully formed, and in some cases there appeared to be facial blends (i.e., the top half of the face displayed a different affective tone from the bottom half of the face). A second difficulty was the brevity of display of each type of expression. In addition, many of these infants appeared to lack underlying muscle tone, with the result that their cheeks and brows exhibited an excessively wrinkled appearance. Absence of a necessary baseline of facial tension for these infants led to great difficulty in determining whether specific components of the face had been activated. Finally, as we indicated earlier, portions of the faces of many of the infants were blocked, or their facial activity was inhibited by the presence of appliances (such as cannulas or gavage tubes) and the tape used to hold these appliances in place. Ultimately, the result was that fewer expressions met criteria and thus fewer overall expressions were coded. It was clear that a substantial amount of facial activity that occurred was not being coded.

\section{EXPERIMENT 2}

In response to the above issues, we designed a revised coding catalog that specifically targeted the problems associated with coding this population of preterm infants (Table 3). Coders who may not be willing to classify a less fully formed expression as a smile may be more readily able to classify such an action as face positive. Similarly, there may be less difficulty in detecting face negative than in differentiating between cry-face, distress brow, or grimace/disgust. The category rhythmic mouth would subsume the previous category, sucking, as well as Trevarthen's (1977) concept of preverbal mouthing. The category nonexpressive, which occurs when the affective tone is neither clearly positive nor clearly negative, replaced the earlier category, relaxed, while the category yawn remained unchanged. Finally, in viewing the tapes, we noticed that a considerable amount of tongue protrusion was displayed, and yet there was no category representing this behavior. It should be noted that even though these revised categories are inductively derived, they require the same coding process as the template-based systems. The facial gestalt is not assessed; instead, criteria in specific regions of the face (e.g., the mouth, eyes, brows, and cheeks) must be met for assigning each category.

\section{Method}

All of the data that had been coded using the previous catalog were discarded. We used the same videotaped sessions that were coded in Experiment 1. The coding procedures were the same as those in the previous experiment (i.e., the same computer interface, the same detection of a behavioral change criterion, the same measures and analyses, etc.); the only difference was the catalog of infant facial activities. Initial testing and refinements of the definitions and coding rules involved only the first (T.H.) and second authors (A.M.). Once a minimum criterion was reached (the median $r(\beta)$ was .86 and the median $\kappa$ was .75 ), an additional coder (A.C.M.) was trained. Over the course of a 3 -month training period, three sets of reliability assessments were performed. Each set involved coding five randomly selected videotaped exams.

\section{Results and Discussion}

Throughout the 3-month training period, the mean and median values for both the $r(\beta)$ and $\kappa$ measures remained above .80 and .60 , respectively. In addition, each of the separate categories demonstrated a relatively equal proportion of agreement, with face positive and tongue protrusion the lowest (.73 and .72, respectively).

Finally, as a point of comparison with the initial coding catalog, the same 12 video segments that had been coded using the initial coding catalog were recoded by T.H. and A.M. (Unfortunately, coder A.C.M. graduated and was not available for this test; however, another undergraduate coder, B.J., was available.) The mean $r(\beta)$ and mean $\kappa$ scores were markedly higher for the revised coding catalog (Table 4). In addition, each of the categories demonstrated a relatively high level of detection with the proportion of agreement ranging from .70 (for face positive) to .96 (for nonexpressive). It is important to note that this catalog demonstrated stability across approximately 8 months (the period of time from the initial tests utilizing coders A.M. and A.C.M., to the last set of tests, which utilized coders A.M. and B.J.). Although coder T.H. had had previous experience with the 12 video segments, approximately 17 months had elapsed since the initial coding, so it is unlikely that prior exposure had an effect. Ideally, we would have preferred using the same coders for both coding systems, but that was impossible. The first pair of coders (M.G. and A.C.) left the project prior to the development of the revised coding system, and, as we indicated, A.C.M. was not available for the current analysis. Although this constitutes a weakness in the experiment, we do not think it is fatal; the first coding system never achieved stability, whereas the revised system was so stable that we were able to easily train an additional coder (B.J.) to the same level of competence as that which we had recorded for A.C.M.

The revised coding categories did not represent a mere collapse of the earlier coding categories, but an overall improvement in the ability of coders to detect the differences between categories. To demonstrate this, the coded files from the initial coding catalog were paired with the coded files from the revised coding catalog. Because each of the coding catalogs used different code numbers for 
Table 3

Revised Coding Categories

\begin{tabular}{|c|c|}
\hline Category & Description of Category \\
\hline face positive & $\begin{array}{l}\text { Included are instances of full or broad smiling. The corners of the mouth are retracted, the cheeks may } \\
\text { show signs of tension, and the eyebrows are either relaxed or raised. The smile may form a broad } \\
\text { grin with the gums or tongue visible, or the lips may be pressed together. Also included: (1) partial } \\
\text { or half smiles (i.e., only one corner of the mouth is retracted and the eyebrows are either relaxed or } \\
\text { raised), and ( } 2 \text { ) face "brightening" (pronounced raising of eyebrows accompanied by a pronounced } \\
\text { opening of the mouth). Not included are cases where the mouth forms a smile, but the eyebrows or } \\
\text { forehead convey a negative affective tone (these cases should be coded face negative). Also excluded } \\
\text { are cases where the face simply conveys a "pleasant" expression resulting from activity in the cheeks, } \\
\text { eyebrows, or forehead (these cases should be coded nonexpressive). }\end{array}$ \\
\hline
\end{tabular}

face negative Included are instances of crying and other types of distress face (frowning, grimacing, or disgust). Each of these expressions is characterized by marked tension in the mouth, cheeks, eyebrows, and forehead. By definition, the disgust face will be accompanied by tongue activity, including tongue protrusions. Also included are cases where there is tension in the mouth, but the mouth expression does not convey a negative tone (indeed, it may look like a smile), and the cheeks, eyebrows, and forehead display an unmistakably negative tone. Not included: (1) instances where a negative expression of the cheeks, eyebrows, or forehead occur without any tension in the mouth; (2) instances where negative tension in the mouth occurs without tension in cheeks, eyebrows, or forehead; or (3) very slight or brief negatively toned twitches or tics.

yawn A yawn begins at the time the mouth begins to widen in an oval manner. The yawn behavior should not include preceding, codable, mouth movements (e.g., tongue protrusion or rhythmic mouth), or instances where the mouth opens but remains relatively stationary, or moves in a horizontal direction. The yawn concludes with the closing of the mouth (again, do not include mouth or face activities that occur immediately after the closing of the mouth). A yawn may be accompanied by a slight raising of the chest and the lower extremities.

rhythmic mouth More than one consecutive (within 2 sec) occurrence of the same type of mouth activity (i.e., includes tongue or lips) during a time in which the remainder of the face is not displaying any codable affective activity. Included are rhythmical activities such as mouthing, lip smacking, chewing, or sucking. Excluded are (1) single occurrences of some type of mouth activity (e.g., wide-open mouth), (2) cases where the occurrences of the same type of movement of the mouth are separated by more than $2 \mathrm{sec}$ of nonactivity of the mouth, or (3) cases of mouth activity composed of different types of mouth movement.

tongue protrusion A tongue protrusion must meet the following criteria: (1) It is a single event or a series of protrusions with less then a 2 -sec pause, (2) the tongue is clearly extended beyond the lips (not just visible in the mouth), and (3) there is no other codable facial affect. The onset should be marked at the time the tongue begins to show a visible extension forward (from a baseline state). The offset occurs when the tongue appears to be retracted into the mouth.

nonexpressive Events that fall into one of the following five groups: (1) There is little or no tension in the face (i.e., there is little or no movement in the eyebrows, forehead, cheeks, and mouth); (2) the affective tone is neither clearly positive nor clearly negative (i.e., expressions that do not meet the criterion for inclusion in face positive or face negative); (3) extremely brief or poorly articulated expressions; (4) single, isolated mouth movements (not including tongue protrusion); (5) bouts of mouth activity composed of different types of mouth movement (i.e., where the action would not be considered rhythmic mouth).

similar categories, and in some cases codes needed to be collapsed, all of the coded files from the initial coding catalog were modified so that they would be comparable to the files from the revised coding catalog. The three categories of cry-face, distress, and grimace/disgust were subsumed under one code (using the same code number as the revised category face negative), and mouthing and sucking were subsumed under one code (using the same code number as the revised category rhythmic mouth). Smile, yawn, and relaxed face were renumbered so that they agreed with the code numbers used with the revised categories (face positive, yawn, and nonexpressive, respectively). All 12 video segments were analyzed in exactly the same manner as any other code/recode comparison; the mean $\kappa$ was only .55 . If the revised coding catalog merely represented a collapse of the initial coding catalog, then the mean $\kappa$ should have been comparable to that observed in the revised coding catalog; this was not the case.

Table 4

Mean $\boldsymbol{r}(\beta)$ and $\kappa$ Scores for the Revised Coding Catalog

\begin{tabular}{|c|c|c|c|c|c|c|c|c|}
\hline & \multicolumn{2}{|c|}{ A.M./B.J. } & \multicolumn{2}{|c|}{ A.M./T.H. } & \multicolumn{2}{|c|}{ B.J./T.H. } & \multicolumn{2}{|c|}{ Totals } \\
\hline & $r(\beta)$ & $\kappa$ & $r(\beta)$ & $\kappa$ & $r(\beta)$ & $\kappa$ & $r(\beta)$ & $\kappa$ \\
\hline$N$ of Cases & 12 & 12 & 12 & 12 & 12 & 12 & 12 & 12 \\
\hline$M$ & .94 & .86 & .93 & .78 & .88 & .77 & .92 & .80 \\
\hline Mdn & .97 & .87 & .93 & .80 & .91 & .79 & .94 & .82 \\
\hline$S D$ & .08 & .08 & .05 & .07 & .06 & .09 & .06 & .08 \\
\hline Min & .73 & .70 & .81 & .63 & .72 & .60 & .75 & .64 \\
\hline $\operatorname{Max}$ & 1.00 & .97 & 1.00 & .89 & .94 & .90 & .98 & .92 \\
\hline
\end{tabular}




\section{GENERAL DISCUSSION}

In general, the results suggest that there may be limits to using inductively derived categories of facial activity that have been developed for use with normal, full-term populations with at-risk populations. It was unfortunate that circumstances prohibited us from using the same individual coders for both coding schemes; thus we cannot say conclusively that the problems we encountered were a result of the first coding scheme. What we can say is that a broader set of inductively derived categories enabled reliable classification of the more global features of the facial activity of infants younger than 40 weeks postconceptional age, including infants who were judged to be at high biological risk.

Although described in broader detail, the categories represent actions that can be analyzed using time-based quantitative strategies, enabling exploration of the preterm infants' early social behavior. Individually, these categories allow us to examine the co-occurrent or sequential placement of facial actions in relation to other infant behaviors, such as gaze, limb, or other motor actions. The knowledge of individual organization provides an empirical foundation for combining facial actions with other infant actions to form global states of activity. The global states can be used to test questions arising from attempts to manipulate the early social experience of these infants, or to examine basic questions related to the early sociocommunicative capabilities of these infants.

\section{REFERENCES}

Bakeman, R., \& Brown, J. V. (1980). Analyzing behavioral sequences: Differences between preterm and full-term infant-mother dyads during the first months of life. In D. B. Swain, R. C Hawkins, L. O. Walker, \& J. H. Penticuff (Eds.), Exceptional infant (Vol. 4, pp. 271-299). New York: Brunner-Mazel.

Beckwith, L., Cohen, S. E., Kopp, C. B., Parmelee, A. H., \& Marcy, T. G. (1976). Caregiver-infant interaction and early cognitive development in preterm infants. Child Development, 47, 579-587.

BocCiA, M., \& CAMPOS, J. J. (1989). Maternal emotional signals, social referencing, and infant's reactions to strangers. In N. Eisenberg (Ed.), New directions for child development (Vol. 44, pp. 25-49). San Francisco: Jossey-Bass.

Brazelton, T. B., Koslowski, B., \& Main, M. (1974). The origins of reciprocity: The early mother-infant interaction. In M. Lewis \& L. Rosenblum (Eds.), The effect of the infant on its caregiver (pp. 4977). New York. Wiley-Interscience.

Campos, J., Barrett, K., Lamb, M., Goldsmith, H., \& Sternberg, C. (1983). Socioemotional development. In P. Mussen (Series Ed.) \& J. J. Campos \& M. H. Haith (Vol. Eds.), Handbook of child psychology: Vol. 2 Infancy and developmental psychobiology (pp. 783915). New York: Wiley.

COHEN, J. (1960). A coefficient of agreement for nominal scales. $E d-$ ucational \& Psychological Measurement, 20, 37-46.

ECKerman, C. O., \& OeHLER, J. M. (1992). Very-low-birthweight newborns and parents as early social partners. In S. L. Friedman \& M. D Sigman (Eds.), The psychological development of low birthweight children. Norwood, NJ: Ablex.

Eckerman, C. O., Oehler, J. M., Hannan, T. E., \& Molitor, A. E. (1995). The development prior to term age of very-prematurelyborn newborns' responsiveness in face exchanges. Infant Behavior \& Development, 18, 283-298.

Eckerman, C. O., Oehler, J. M., Medvin, M. B., \& Hannan, T. E. (1994). Premature newborns' responsiveness to human speech and touch before term age. Infant Behavior \& Development, 17, 55-70. Eckman, P., \& FriesEN, W. V. (1978). The facial action coding system (FACS). Palo Alto: Consulting Psychologists Press.

Field, T. M., Vega-Lahr, N., Goldstein, S., \& Scafidi, F. (1987). Face-to-face interaction behavior across early infancy. Infant Behavior \& Development, 10, 111-116.

Field, T. M., Woodson, R., Cohen, D., Greenberg, R., Garcia, R., \& Collins, K. (1983). Discrimination and imitation of facial expressions by term and preterm neonates. Infant Behavior \& Development, 6, 485-489.

FoGEL, A. (1979). Peer vs. mother directed behavior in 1- to 3-monthold infants. Infant Behavior \& Development, 2, 189-200.

FoGEL, A. (1981). The ontogeny of gestural communication: The first six months. In R. Stark (Ed.), Language behavior in infancy and early childhood (pp. 17-44). New York: Elsevier, North-Holland.

Fogel, A., \& Hannan, T. E. (1985). Manual actions of 9- to 15-weekold human infants during face-to-face interaction with their mothers. Child Development, 56, 1271-1279.

HanNan, T. E., \& FoGEl, A. (1987). A case-study assessment of 'pointing' during the first 3 months of life. Perceptual \& Motor Skills, 65, 187-194.

HolLenbeCK, A. R. (1978). Problems of reliability in observational research. In G. P. Sackett (Ed.), Observing behavior, Volume 2: Data collection and analysis methods (pp. 79-98). Baltimore: University Park Press.

HollingsheAD, A. B. (1957). Two-factor index of social position. Unpublished manuscript, Yale University.

IZARD, C. E. (1979). The maximally discriminative facial movement coding system $(M A X)$. Newark, DE: Instructional Resources Center.

IZARD, C. E., \& DOUgherTY, L. M. (1980). A system for identifying affect expressions by holistic judgments (AFFEX). Newark, DE: Instructional Resources Center.

KAYE, K. (1980). Estimatıng false alarms and missed events from interobserver agreement: A rationale. Psychological Bulletin, 88, 458-468.

KAYE, K., \& FoGEL, A. (1980). The temporal structure of face-to-face communication between mothers and infants. Developmental Psychology, 16, 454-464.

KIENAPPLE, K. (1987). Micro-analytic data analysis package. Behavior Research Methods, Instruments, \& Computers, 19, 335-337.

KRAEMER, H. C. (1982). Estimating false alarms and missed events from interobserver agreement: Comment on Kaye. Psychological Bulletin, 92, 749-754.

Malatesta, C. Z., Grigoryev, P., Lamb, C., Albin, M., \& Culver, C. (1986). Emotion socialization and expressive development in preterm and full term infants. Child Development, 57, 316-330.

Malatesta, C. Z., Tesman, J. R., \& Shepard, B. (1990, April). Facial and vocal expressiveness in fullterm and preterm children. Paper presented at the Seventh International Conference on Infant Studies, Montreal.

Minde, K., Perrotta, M., \& Marton, P. (1985). Maternal caretaking and play with fullterm and preterm infants. Journal of Child Psychology \& Psychiatry, 26, 231-244

Minde, K., Whitelaw, A., Brown, J., \& Fitzhardinge, P. (1983). Effect of neonatal complications in premature infants on early parentinfant interactions. Developmental Medicine \& Clinical Neurology, 25, 763-775.

Oehler, J. M., Eckerman, C. O., \& Wilson, W. H. (1988). Social stimulation and the regulation of premature infants' state prior to term age. Infant Behavior \& Development, 11, 333-351.

OSTER, H. (1978). Facial expression and affect development. In M. Lewis \& L. A. Rosenblum (Eds.), The development of affect (pp 43-76). New York: Plenum.

OSTER, H., \& Rosenstein, D. (in press). Baby FACS: Analyzing facial movements in infants Palo Alto: Consultıng Psychologists Press.

Trevarthen, C. (1977). Descriptive analysis of infant communicative behavior. In H. R. Schaffer (Ed.), Studies in mother-infant interaction (pp. 227-270). London: Academic Press.

Triangle Research Collaborative. (1985). Observational coding system (OCS) tools. Research Triangle Park, NC: Author.

(Manuscript received March 16, 1994; revision accepted for publication March 15, 1995.) 